\title{
Canine Femoral Neck Lengthening Combined with DARthroplasty to Manage Severe Canine Juvenile Hip Dysplasia: A Case Report
}

\author{
Massimo Petazzoni ${ }^{1}$ Melania Dallago ${ }^{2}$ \\ ${ }^{1}$ Clinica Veterinaria Milano Sud, Peschiera Borromeo, Milano, Italy \\ ${ }^{2}$ Clinica Veterinaria Zoolife, Mezzolombardo, Trento, Italy \\ VCOT Open 2019;2:e25-e29.
}

Address for correspondence Melania Dallago, DVM, Clinica Veterinaria Zoolife, Mezzolombardo, Via Campi Neri 35, Cles 38023, Trento, Italy (e-mail: meld_88@hotmail.com).

\begin{abstract}
Keywords

- hip dysplasia

- femoral neck lengthening

- dorsal acetabular rim

- arthroplasty

- canine

Objective This study is a case description of femoral neck lengthening and dorsal acetabular rim (DAR) arthroplasty to treat severe hip luxation in a 5-month-old male Cocker Spaniel $(14.3 \mathrm{~kg})$ and to compare the clinical outcome with that of contralateral femoral neck excision.

Study design Case report.

Results Femoral head coverage improved after surgery (preoperative lateralization of the centre of the femoral head with regard to the dorsolateral acetabular rim: $10 \mathrm{~mm}$; postoperative lateralization of the centre of the femoral head: $-1.3 \mathrm{~mm}$ ). The postoperative Barden's test was negative. The dog was bearing weight 2 weeks postoperatively. Radiographic signs of bone healing were present at the 6-week follow-up examination. No complications were detected.

Conclusions Femoral neck lengthening and DAR augmentation were performed in a growing dog affected by severe hip luxation and DAR insufficiency.
\end{abstract}

\section{Introduction}

Canine hip dysplasia is one of the most common orthopaedic conditions in the $\operatorname{dog}^{1,2}$ It is generally described as a developmental disorder, characterized by varying degrees of laxity of the hip joint, which leads to continued femoral head subluxation, shallow acetabulum, remodelling of the joint surfaces, osteoarthritis and pain. ${ }^{1,2}$

This case report describes the surgical procedure and the clinical outcomes of unilateral femoral neck lengthening ${ }^{3}$ combined with dorsal acetabular rim (DAR) augmentation, ${ }^{4}$ performed in a juvenile dog affected by severe hip joint laxity. Clinical outcome was compared with contralateral femoral neck excision.

\section{Case Report}

A 5-month-old male Cocker Spaniel (14.3 kg) was admitted because of chronic, bilateral pelvic-limb lameness. The owner

received

December 22, 2018

accepted after revision

June 25, 2019 reported that the dog showed bunny hopping motion, reluctance to walk and difficulties in rising after rest.

\section{Pre-Clinical Assessment}

On clinical examination, the dog was reluctant to walk. Significant signs of discomfort were elicited upon hip manipulation and extension of both hips caused pain. Bilateral decreased hind-limb muscle volume was noted. Barden's test was performed under sedation: lateral translation was visually estimated being greater than $10 \mathrm{~mm}$. Hips range of motion was assessed with the aid of a plastic goniometer and measured $50^{\circ}$ in flexion and $160^{\circ}$ in extension bilaterally. ${ }^{5}$ Clinical, orthopaedic and neurological examinations were otherwise unremarkable.

\section{Diagnostic Imaging}

The dog underwent radiographic survey under general anaesthesia. A ventrodorsal hip-extended radiograph was taken ( - Fig. 1A). This radiograph further corroborated the severe

\footnotetext{
(c) 2019 Georg Thieme Verlag KG Stuttgart · New York
}

License terms

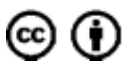
DOI https://doi.org/
10.1055/s-0039-1694036.
ISSN 2625-2325. 


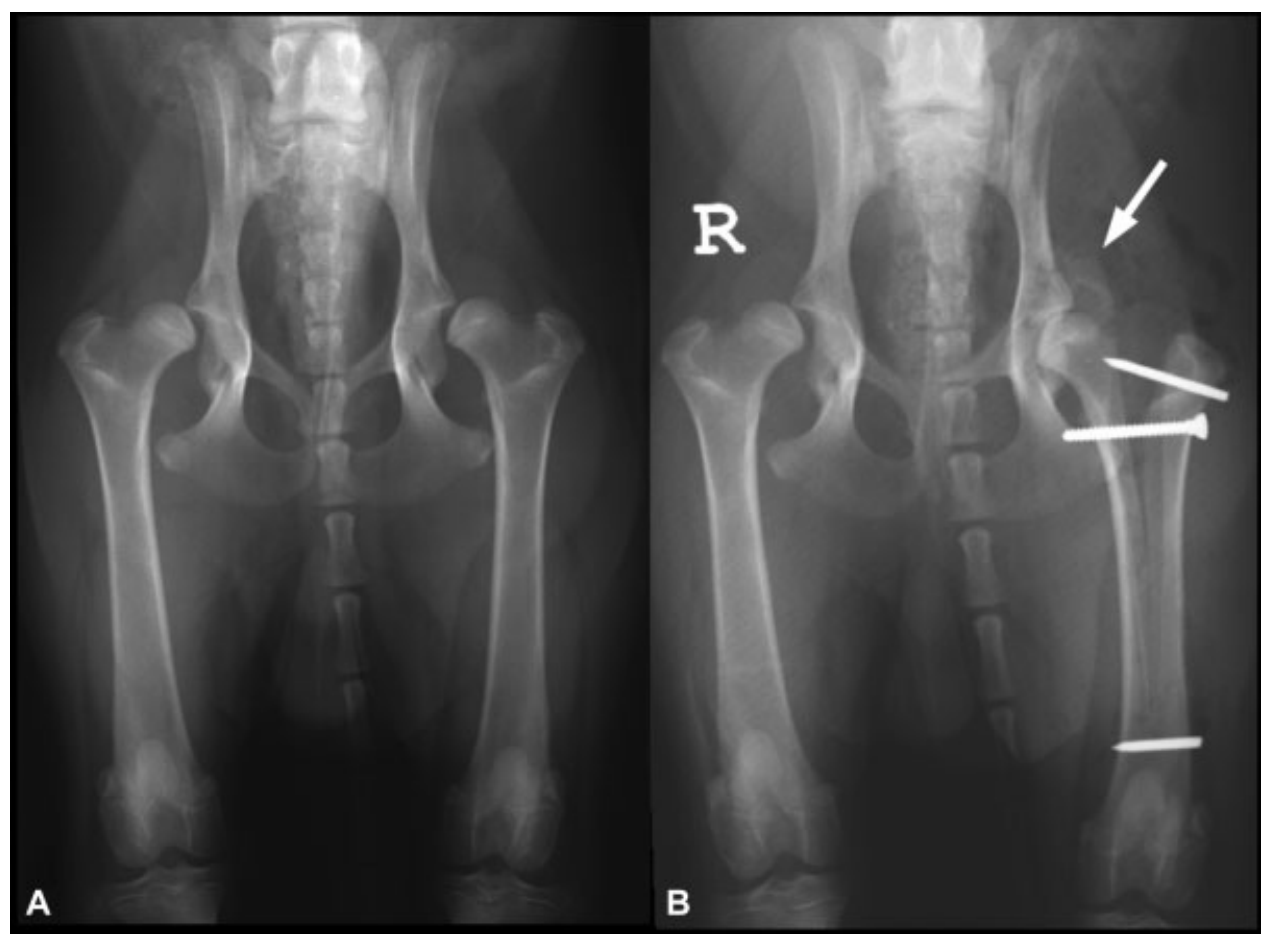

Fig. 1 (A) Preoperative ventrodorsal hip extended projection. Severe bilateral femoral head dislocation combined with shallow acetabulum. (B) Postoperative ventrodorsal hip view showing reduction in the left femoral head, improved hip joint congruity, and corticocancellous bone-graft strips (white arrow).

joint laxity of both hips, evidenced by lateral displacement of the femoral heads. Radiographs also showed a shallow acetabulum, the presence of a subtle circumferential femoral head osteophyte and the absence of other signs of osteoarthritis.

A commercially available computer programme (Osirix v.2.7.5 32-bit; Pixmeo SARL, Bernex, Switzerland) was used to determine the Norberg angle and to measure the lateralization of the centres of the femoral heads with regard to the dorsolateral acetabular rims. ${ }^{6}$ Norberg angle was $38^{\circ}$ for the left hip and $46^{\circ}$ for the right one (normal value $\left.>105^{\circ}\right){ }^{7}$ The lateralization of the centre of the femoral head was $10 \mathrm{~mm}$ bilaterally (normal value $\leq 0 \mathrm{~mm}$. In a normal hip the centre of the femoral head should lie just under or medial the dorsolateral acetabular edge). ${ }^{6}$

\section{Surgical Technique}

Informed consent was obtained from the owner for femoral neck lengthening and DAR augmentation.

\section{Femoral Neck Lengthening}

The dog was positioned in lateral recumbency with the left hind limb uppermost. A craniolateral approach was taken to access the left hip. ${ }^{8}$ The origin of the vastus lateralis muscle was detached to approach the proximal femur. The remnants of the quadriceps muscle group and the cranial belly of the sartorius muscle were moved medially using a Hohmann retractor.

A 3.5-mm hole was drilled at the level of the desired end of the osteotomy distally, in the sagittal plane, $15 \mathrm{~mm}$ proximal to the patella with the stifle in extension. This hole served as a marker and as an osteotomy stopper.
The osteotomy was performed using an oscillating saw starting between femoral neck and the greater trochanter, medial to the insertion of the obturator and gemelli muscles. The osteotomy was extended distally, centred on the femoral shaft, and ending on the predrilled hole. A $2 \mathrm{~mm}$ threaded pin was inserted transversely just distal to the predrilled hole to prevent further distal femoral splitting. The pin was then cut flush at the level of the lateral cortex.

At the level of the distal aspect of the greater trochanter, a $2.5-\mathrm{mm}$ hole was drilled in the lateral cortex, perpendicular to the femoral axis, up to the level of the osteotomy aiming at the lesser trochanter. A $3.5-\mathrm{mm}$ self-tapping cortical screw (3.5-mm self-tapping cortical screw; Gruppo Bioimpianti, Peschiera Borromeo, Italy) was then inserted. As the tip of this screw reached the trabecular bone medial to the osteotomy, its subsequent advancement forced the greater trochanter to move laterally along the screw thread. Distraction was performed slowly at a rate of $1 \mathrm{~mm}$ distraction per minute, measured at the proximal split bone. Multiple Barden's tests were performed intraoperatively until the lateral transitional motion of the femoral head stopped.

A positional 2-mm threaded Kirschner wire was inserted through the lateral cortex of the greater trochanter, $1 \mathrm{~cm}$ distal to its proximal edge, to maintain the trochanteric lateralization. The $3.5-\mathrm{mm}$ splitting screw was removed and its $2.5 \mathrm{~mm}$ hole was completed threading a 2.5 tap hole in the trans cortex. The length of the hole was measured using a depth gauge and a 3.5 cortical screw $4 \mathrm{~mm}$ longer than the measured length was inserted. A 12-mm left femoral neck lengthening wedge (Femoral neck lengthening Slocum plastic wedges; Slocum Enterprises, Eugene, Oregon, 
United States) was placed into the osteotomy, to maintain neck lengthening.

\section{DARrthroplasty|Augmentation}

The iliac crest was approached as previously described. ${ }^{2,9}$ The DAR procedure was performed as described by Slocum and Slocum. ${ }^{4}$

Before the surgical wound was closed, a sponge soaked with dibotermin alfa (TruScient; Pfizer Italia Srl, Rome, Italy) $(0.66 \mathrm{mg})$ was cut into four stripes and applied on the DAR grafts, and on the proximal, mid and distal femoral diaphysis to promote new bone formation.

The vastus lateralis muscle was attached to the articular capsule proximally. Soft tissue layers were closed as per routine. ${ }^{8}$

\section{Postoperative Management}

Postoperative radiographs (ventrodorsal hip-extended and mediolateral projection of the femur) were taken ( - Fig. 1B). The lateralization of the centre of the femoral head was 1.3 $\mathrm{mm}$. The osteotomy was delineated and ended at the planned level of the distal femur. The threaded pins and proximal screw were in their planned positions.

Postoperative medications included firocoxib (Previcox; Merial Italia SPA, Milano, Italy) $(5 \mathrm{mg} / \mathrm{kg}$ once daily for 3 days), amoxicillin-clavulanic acid (Synulox; Zoetis Italia S.r.l. Rome, Italy) (20 mg/kg twice daily for 10 days) and tramadol (Altadol; Formevet S.r.l., Milano, Italy) $(2 \mathrm{mg} / \mathrm{kg}$ twice daily for 10 days). Cage rest was enforced, and exercise restricted to short daily leash walks for a period of 8 weeks. Physiotherapy was proposed but not performed (owner's economic limitation).

\section{Postoperative Assessment}

Follow-up examinations were performed at 2, 3, 4, 5, 6 and 8 weeks and at 2 and 7 years after surgery. At each follow-up examination, the presence of lameness, range of motion and pain upon joint flexion and extension were assessed and a ventrodorsal hip-extended radiograph was taken.
At 2 weeks after surgery, the dog started to bear weight consistently on the left pelvic limb. Lameness was difficult to assess in the absence of full weight-bearing on the luxated right pelvic limb. Lameness and pain upon manipulation of the left hip were absent from week 3. Joint motion was unchanged during the recovery period.

The position of the implant remained stable during the first 2 months. At the 2-year follow-up, the distal threaded pin was seen to have migrated laterally subcutaneously, causing mild discomfort on palpation of the distal and lateral aspects of the femur. The pin was removed under general anaesthesia.

At 3 weeks after surgery, there were radiographic signs of bone healing, with new bone formation bridging different parts of the osteotomy, especially where the dibotermin alfa was sited (-Fig. 2). Trabecular bone was clearly detectable from week 6 , at which time the proximal and distal parts of the osteotomy were healed.

Five weeks after surgery the dog underwent excision arthroplasty on the right pelvic limb (-Fig. 3A). At 8 weeks, a subtle radiolucent fissure line was present underneath the positional screw. A prominent callus on the bone grafts used for DAR augmentation was present since week 5 . Remodelling of the latter led to the formation of an adequate acetabular roof (-Fig. 2).

At the 2-year and 7-year follow-up examinations, the owner reported that the animal had normal gait on both pelvic limbs. At clinical examination, no lameness of the pelvic limbs was detected. Range of motion of the right hip was normal in flexion and mildly limited in extension $\left(50-145^{\circ}\right)$, whereas the range of motion of the left hip was normal $\left(50-160^{\circ}\right) .^{5}$ Muscle volume discrepancy was detected radiographically at the 2year and at the 7-year follow-up (-Figs. 3B and 4).

\section{Discussion}

Common preventive surgeries acting on the DAR may be ineffective in puppies with a high degree of laxity and shallow acetabulum, and such animals, as our case, should be considered candidates for excision arthroplasty. ${ }^{10}$

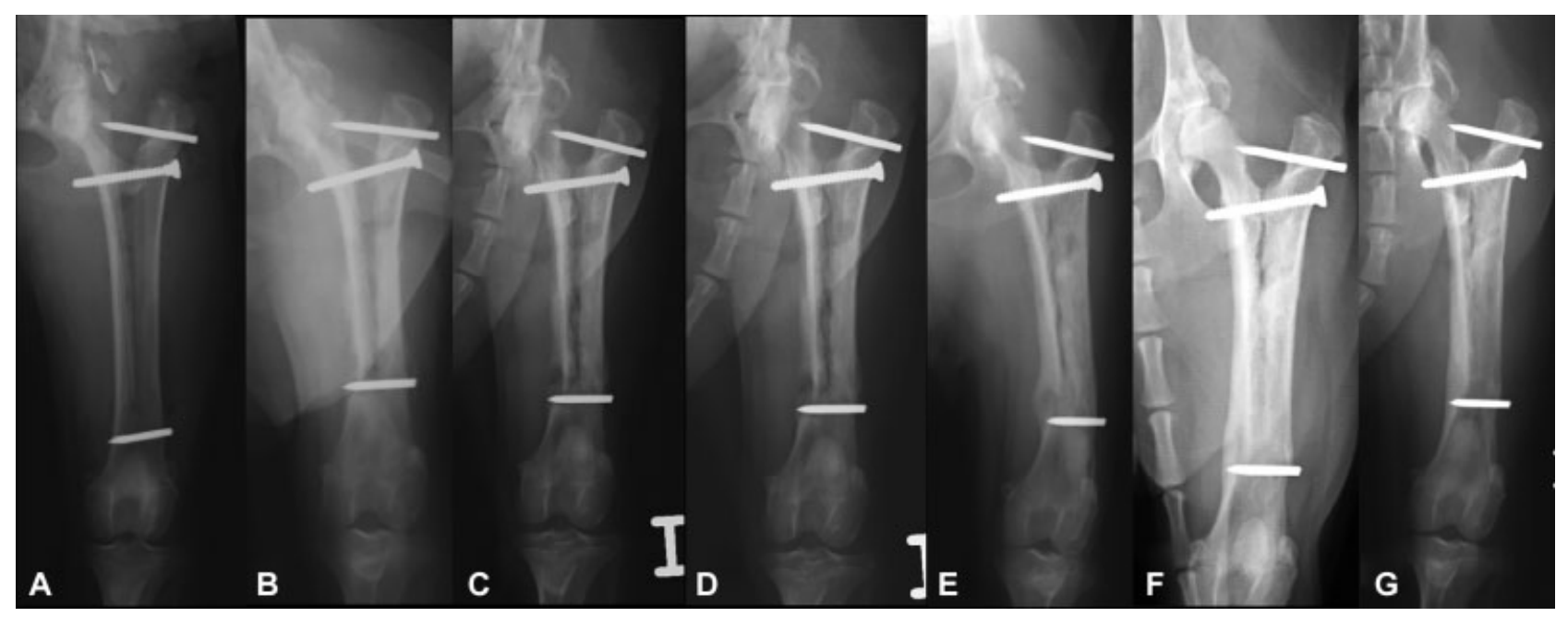

Fig. 2 Femur, cranial-caudal radiographs: immediately postoperatively (A) and at 2 weeks (B), at 3 weeks (C), at 4 weeks (D), at 5 weeks (E), 6 weeks (F), and at 8 weeks $(G)$ after surgery. 


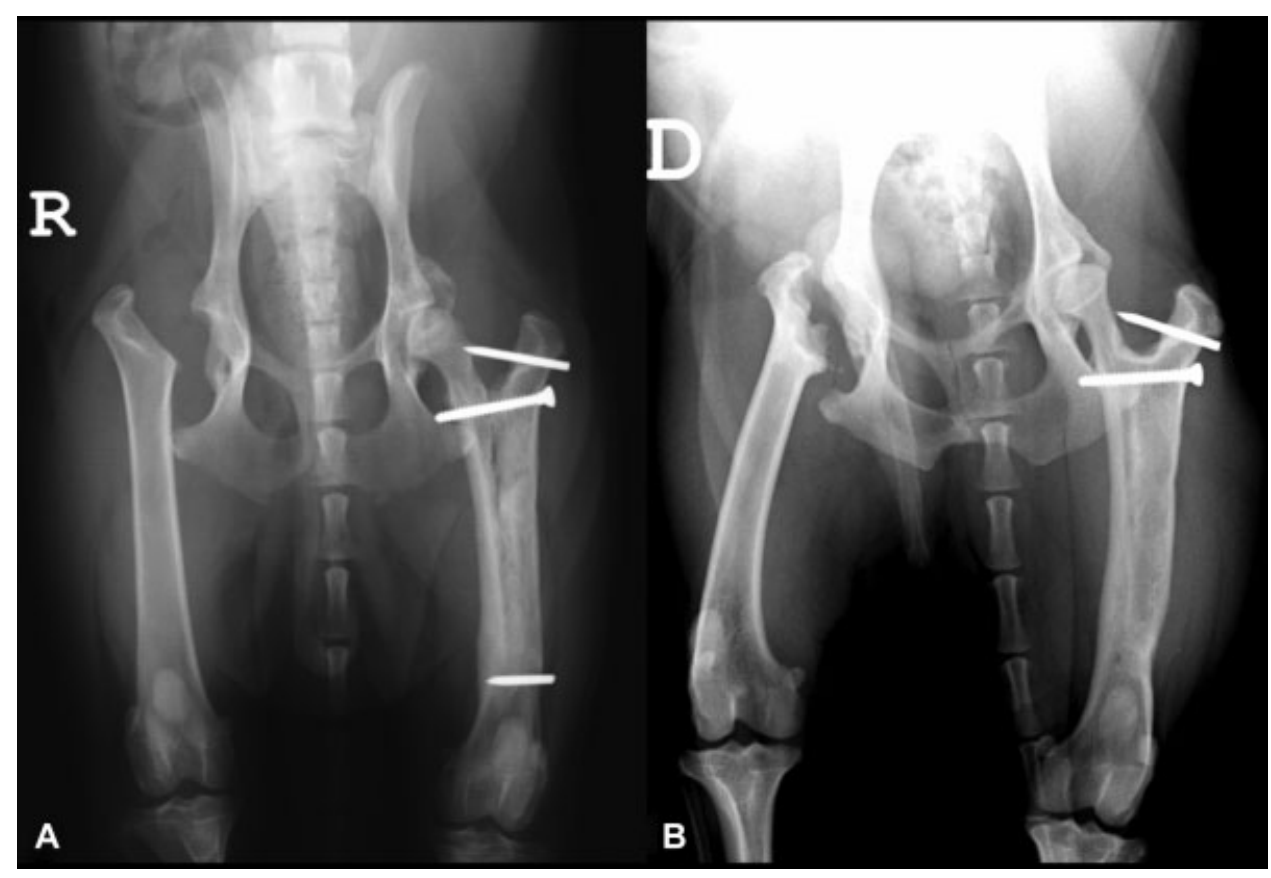

Fig. 3 (A) Postoperative radiograph of femoral head and neck excision. (B) Radiographic follow-up at 2 years. Note the progression of bone remodelling of the left femur and the dorsal acetabular rim shaping. No radiographic signs of progression of osteoarthritis. The distal threaded Kirschner wire was removed.

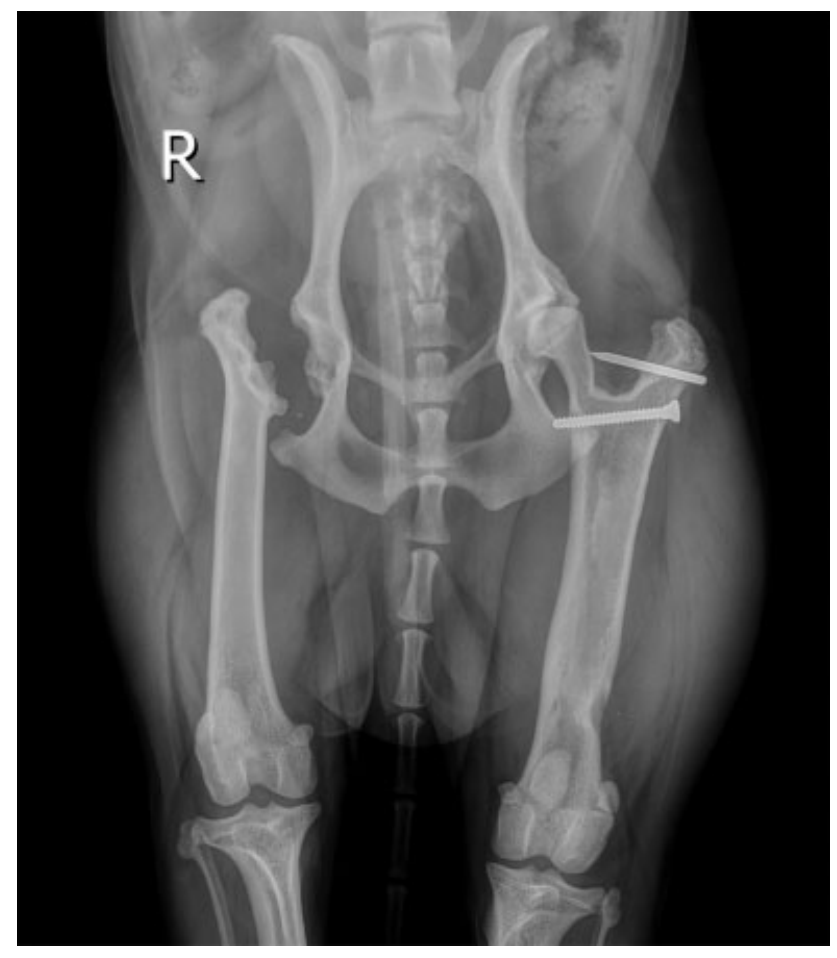

Fig. 4 Radiographic follow-up at 7 years. Note the discrepancy in muscle volume.

Two biomechanical terms need to be elucidated to understand femoral neck lengthening. The first is the femoral neck length, defined as the distance between the medial limit of the femoral head and the lateral limit of the greater trochanter. The second is the moment, determined by multiplying force by distance. Gluteal muscles (internal hip rotator muscles) and the obturator muscles (external hip rotator muscles) are attached to the greater trochanter. These muscles together exercise the same amplitude of moment on the femoral head, providing joint stability. If the femoral neck length increases, the femoral head approaches the acetabulum, and internal and external hip rotator muscle forces increase equally, generating a higher moment acting on the hip joint.,

The surgical procedure, consisting of femoral neck lengthening and DAR augmentation, ameliorated hip laxity and achieved greater acetabular coverage of the femoral head immediately after surgery. The substantial difference between the preoperative lateralization of the centre of the femoral head $(10 \mathrm{~mm})$ and postoperative lateralization of the centre of the femoral head $(-1.3 \mathrm{~mm})$ was an objective demonstration of the reduced joint laxity, with the negative intraoperative Barden's test enforcing this latter finding.

To maintain the femoral distraction, femoral neck lengthening Slocum plastic wedges were used. Since Slocum Enterprises has closed, other types of wedges should be considered to avoid collapse of the femoral neck. Tibial tuberosity advancement wedges used in cranial cruciate ligament rupture surgery could be used instead (Titanium cages [Titanium cage, Kyon, Zurich, Switzerland; Titanium cage, Rita Leibinger Medical, Mühlheim an der Donau, Germany], Porous titanium wedges [Porous titanium wedge, Orthomed, Huddersfield, United Kingdom; Porous titanium wedge, Ad Maiora srl, Cavriago, Italy).

Acceptable limb function was noted 2 weeks after surgery, when the dog, which had been reluctant to move, became able to walk using the operated limb. A good level of comfort was reached with traditional postoperative care, and any sign of discomfort disappeared from the third week postoperatively. The absence of femoral head subluxation was probably consistent with the absence of capsular stretching. The surgical intervention successfully restrained the progression 
of osteoarthritis. The degenerative changes seen on the preoperative and postoperative radiographs were essentially the same, being confined to thin, circumferential femoral head increased radio-opacity, probably attributable to a peripheral osteophyte.

Five weeks after surgery, the dog underwent femoral head and neck excision on the contralateral right hip because of financial reasons. At the 2-year follow-up no lameness was detected in either limb, although the muscle volume between the two was markedly different, in favour of the limb that underwent femoral neck lengthening and DAR augmentation. The assessment of lameness and muscle development was subjective and thus of questionable value. The use of force plate analysis and thigh circumference measurement would have given an objective evaluation.

In our experience, this operation was not more difficult than other preventive procedures comprising osteotomies. We believe distraction of the proximal femoral shaft was the most important and delicate step of the femoral neck lengthening and must be performed gently to prevent distal femoral fractures. A transverse threaded pin or screw placed distal and perpendicular to the osteotomy may avoid further distal splitting into the stifle joint.

A bone gap resulting from the femoral splitting could potentially lead to delayed union or non-union. However, fast new bone formation in immature dogs might make this less likely. The application of dibotermin alfa might have promoted the bone healing process. This could be supported by 5- and 6week postoperative radiographs, which showed that the sites where dibotermin alfa had been applied were bridging earlier by new bone. The proximal screw may also have contributed to promote stability, thereby stimulating healing.

Implant loosening (migration of the distal threaded Kirschner wire) occurred in the presence of completed bone healing, without creating clinical concerns. Implant failure must, however, be considered as an important postoperative complication.

In conclusion, femoral neck lengthening and DAR augmentation were performed in a growing dog with severe hip luxation and DAR insufficiency. This surgical intervention provided adequate femoral head coverage and the dog maintained full joint function over 7 years. No major complications have been detected. The femoral head and neck excision performed on the contralateral pelvic limb could be considered for the purposes of comparison. Although outcomes were assessed subjectively by one surgeon, the later- alization of the centre of the femoral heads was evaluated objectively. At the 2-year and at 7-year follow-up examinations, outcomes in the hip with preserved hip anatomy were better.

Author Contribution

Massimo Petazzoni performed the surgery and revised the current manuscript.

Melania Dallago wrote the manuscript and submitted it to vCOTOpen.

Conflict of Interest

None declared.

\section{References}

1 Henricson B, Norberg I, Olsson SE. On the etiology and pathogenesis of hip dysplasia: a comparative review. J Small Anim Pract 1966;7(11):673-688

2 Piermattei DL, Flo G, DeCamp C. The hip joint. In: Piermattei D, Flo G, DeCamp C, eds. Handbook of Small Animal Orthopedics and Fracture Repair. Missouri: Elsevier Saunders; 2006:461-511

3 Devine T, Slocum B. Results of femoral neck lengthening procedure in 75 dogs. Paper presented at: 22nd Annual Meeting Veterinary Orthopedic Society; Whistler, Canada; March 4-11, 1995:3

4 Slocum B, Slocum T. The hip joint. In: Bojrab MJ, ed. Current Techniques in Small Animal Surgery. Baltimore: Williams \& Wilkins; 1998:1168-1170

5 Jaegger G, Marcellin-Little DJ, Levine D. Reliability of goniometry in Labrador Retrievers. Am J Vet Res 2002;63(07):979-986

6 Vezzoni A, Dravelli G, Vezzoni L, et al. Comparison of conservative management and juvenile pubic symphysiodesis in the early treatment of canine hip dysplasia. Vet Comp Orthop Traumatol 2008;21(03):267-279

7 Douglas SW, Williamson HD. The limb bones and joints. In: Douglass SW, Williamson HD, eds. Veterinary Radiological Interpretation. Philadelphia: Lea \& Febiger; 1970:91-121

8 Piermattei DL, Johnson KA. Approach to the craniodorsal aspect of the hip joint through a craniolateral incision. In: Piermattei DL, Johnson KA, eds. An Atlas of Surgical Approaches to the Bones and Joints of the Dog and the Cat. 4th ed. Philadelphia: Elsevier Saunders; 2004:290-295

9 Piermattei DL, Johnson KA. Approach to the wing of the ilium and dorsal aspect of the sacrum. In: Piermattei DL, Johnson KA, eds. An Atlas of Surgical Approaches to the Bones and Joints of the Dog and the Cat. 4th ed. Philadelphia: Elsevier Saunders; 2004: 278-281

10 Smith GK, Karbe GT, McDonald-Lynch MB. Pathogenesis, diagnosis, and control of canine hip dysplasia. In: Tobias K, Johnston S, eds. Veterinary Surgery Small Animal. Vol 1. Missouri: Elsevier Saunders; 2012:824-848 